\title{
Bietet Kunden noch mehr Service
}

Auf der neuen Homepage von Miele Professional finden sie jetzt besonders schnell zum passenden Produkt: www.mieleprofessional.de bietet eine übersichtliche Struktur in modernem Layout und mit komfortabler Filterfunktion. Bei Bedarf erhalten potenzielle Käufer dort auch umfassende Informationen über Finanzierung, Zubehör und mehr. Beim Layout wurden auch Kundenanregungen umgesetzt: Mit 1 Klick auf den Reiter „Branchen“ öffnen sich 9 Felder, zum Beispiel für „Medizin“, „Hotel \& Restaurant“ oder „Dienstleistungen \& Handwerk“. Durch Auswahl der gewünschten Branche stehen weiterführende Informationen auf ei-

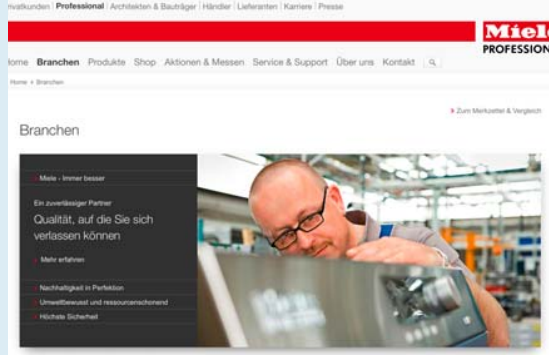

ner neuen Seite zur Verfügung. Über eine Bildleiste im oberen Bereich gelangt der Nutzer dann zur passenden Geräteauswahl, von Wäschereimaschinen über Gewerbegeschirrspüler bis zu Desinfektoren und Sterilisatoren für den Einsatz in Krankenhaus und Labor.Zu den Produkten gibt es unter anderem relevante Informationen über Abmessungen, Programmlaufzeiten, Verbrauchswerte oder den serienmäßigen Lieferumfang. Außerdem können Nutzer verschiedene Produkte speichern und miteinander vergleichen. Wie individuelle Lösungen für unterschiedlichste Kunden aussehen können, ist auf der neuen Homepage unter „Service \& Support“ beschrieben. Hier gibt es Informationen über die Planung neuer Räumlichkeiten oder über verschiedene Wartungs- und Serviceverträge - und Prospekte oder Gebrauchsanweisungen zum kostenlosen Download.

Nach einer Pressemitteilung der Miele \& Cie. KG, Gütersloh 